\title{
A Bayesian Method for the Extinction
}

\author{
Hai-Jun Tian ${ }^{1,2}$, Chao Liu${ }^{1}$, Jing-Yao $\mathrm{Hu}^{1}$, Yang $\mathrm{Xu}^{1}$, Xue-Lei Chen ${ }^{1}$ \\ ${ }^{1}$ National Astronomical Observatories, Chinese Academy of Sciences, Beijing 100012 \\ ${ }^{2}$ China Three Gorges University, Yichang, 443002. Email: hjtian@lamost.org
}

\begin{abstract}
We propose a Bayesian method to measure the total Galactic extinction parameters, $R_{V}$ and $A_{V}$. Validation tests based on the simulated data indicate that the method can achieve the accuracy of around $0.01 \mathrm{mag}$. We apply this method to the SDSS BHB stars in the northern Galactic cap and find that the derived extinctions are highly consistent with those from Schlegel et al. (1998). It suggests that the Bayesian method is promising for the extinction estimation, even the reddening values are close to the observational errors.
\end{abstract}

Keywords. dust, extinction stars: horizontal-branch methods: statistical

\section{Introduction}

The Galactic interstellar extinction is attributed to the absorption and scattering of the interstellar medium, such as gas and dust grains(Draine 2003). The extinction, as a function of the wavelength, is related to the size distribution and abundances of the grains. Therefore, it plays an important role in understanding the nature of the interstellar medium. In addition, the flux of extragalactic objects suffers from different extinction in different bands, which leads to some bias on the extragalactic studies (Tian et al. 2011). Hence, understanding the total interstellar extinction in each line of sight (hereafter los) is crucial for accurate flux measurements.

The all-sky dust map can either be constrained by measuring interstellar extinction, or by employing a tracer of ISM, e.g., HI. One of the most broadly used dust maps was published by Schlegel et al. (1998) (hereafter SFD), who derived it from the dust emission at $100 \mu \mathrm{m}$ and $240 \mu \mathrm{m}$. Since then, many other works have claimed discrepancy with their results(Arce \& Goodman 1999; Guy et al. 2010).

This paper propose an effective method to examine the extinction values of SFD using the BHB stars as tracer in the northern Galactic cap. BHB stars are luminous and far behind the dusty disk, which contribute to most of the interstellar extinction.

\section{Methods}

Bayesian Method for Color Excess. The total Galactic extinction in a given los is measured from the offset of the observed color indexes of the BHB stars from their intrinsic values. A set of BHB stars, which dereddened color indexes, $\left\{\mathbf{c}_{\mathrm{k}}\right\}$ (where $k=$ $\left.1,2, \ldots, N_{B \text { н }}\right)$, are known, are selected as template stars. The reddening of a field BHB stars can then be estimated by comparing their observed colors with the templates. Given a los $i$ with $N_{i}$ field BHB stars, the posterior probability of the reddening $\mathbf{E}_{\mathbf{i}}$ is denoted as $p\left(\mathbf{E}_{\mathbf{i}} \mid\left\{\hat{\mathbf{c}}_{\mathbf{i j}}\right\},\left\{\mathbf{c}_{\mathbf{k}}\right\}\right)$, where $\hat{\mathbf{c}}_{\mathbf{i j}}$ is the observed color index vector of the BHB star $j$ in the los $i$, and $\mathbf{c}_{\mathbf{k}}$ the intrinsic color index vector of the template BHB star $k$. According to the Bayes theorem, this probability can be written as

$$
p\left(\mathbf{E}_{\mathbf{i}} \mid\left\{\hat{\mathbf{c}}_{\mathbf{i j}}\right\},\left\{\mathbf{c}_{\mathbf{k}}\right\}\right)=\mathbf{p}\left(\left\{\hat{\mathbf{c}}_{\mathbf{i j}}\right\} \mid \mathbf{E}_{\mathbf{i}},\left\{\mathbf{c}_{\mathbf{k}}\right\}\right) \mathbf{P}\left(\mathbf{E}_{\mathbf{i}} \mid\left\{\mathbf{c}_{\mathbf{k}}\right\}\right) .
$$



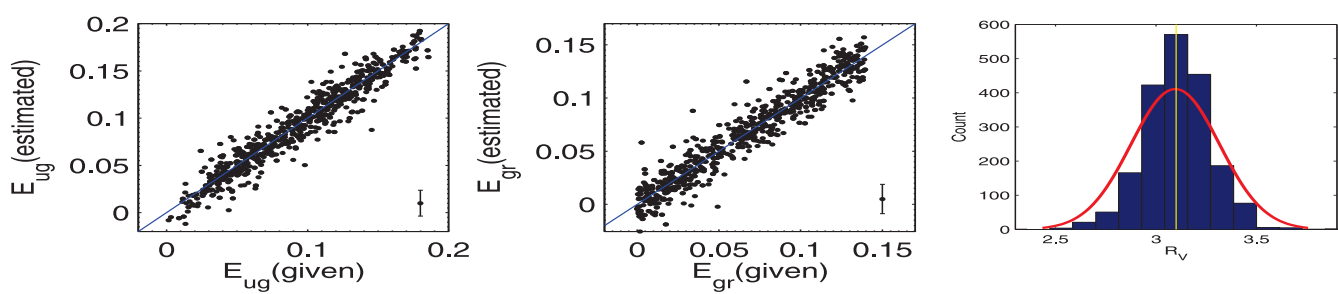

Figure 1. Comparison between the estimated and true values, and the histogram of $R_{V}$.

The right-hand side can now be rewritten as

$$
p\left(\mathbf{E}_{\mathbf{i}} \mid\left\{\hat{\mathbf{c}}_{\mathbf{i j}}\right\},\left\{\mathbf{c}_{\mathbf{k}}\right\}\right)=\prod_{\mathbf{j}=1}^{\mathbf{N}_{\mathbf{i}}} \sum_{\mathbf{k}=\mathbf{1}}^{\mathbf{N}_{\text {B H B }}} \mathbf{p}\left(\hat{\mathbf{c}}_{\mathbf{i j}} \mid \mathbf{E}_{\mathbf{i}}, \mathbf{c}_{\mathbf{k}}\right) \mathbf{p}\left(\mathbf{E}_{\mathbf{i}}\right) .
$$

We assume that the likelihood $p\left(\hat{\mathbf{c}}_{\mathbf{i j}} \mid\left(\mathbf{E}_{\mathbf{i}}, \mathbf{c}_{\mathbf{k}}\right)\right)$ is a multivariate Gaussian:

$$
p\left(\hat{\mathbf{c}}_{i j} \mid \mathbf{E}_{i}, \mathbf{c}_{\mathbf{k}}\right)=\frac{\mathbf{1}}{(\mathbf{2} \pi|\mathbf{\Sigma}|)^{\mathbf{m} / \mathbf{2}}} \exp \left(-\mathbf{x}^{\mathbf{T}} \boldsymbol{\Sigma}^{-1} \mathbf{x}\right)
$$

where $\mathbf{x}=\mathbf{E}+\mathbf{c}_{k}-\hat{\mathbf{c}}_{i j}$, and $\boldsymbol{\Sigma}$ is the $\mathrm{m} \times \mathrm{m}$ covariance matrix of the measurement of the color indexes of the star $j$,

$$
\boldsymbol{\Sigma}=\left[\begin{array}{cccc}
\sigma_{u}^{2}+\sigma_{g}^{2} & -\sigma_{g}^{2} & 0 & 0 \\
-\sigma_{g}^{2} & \sigma_{g}^{2}+\sigma_{r}^{2} & -\sigma_{r}^{2} & 0 \\
0 & -\sigma_{r}^{2} & \sigma_{r}^{2}+\sigma_{i}^{2} & -\sigma_{i}^{2} \\
0 & 0 & -\sigma_{i}^{2} & \sigma_{i}^{2}+\sigma_{z}^{2}
\end{array}\right]
$$

where the $\sigma_{u}, \sigma_{g}, \sigma_{r}, \sigma_{i}$, and $\sigma_{z}$ are the measurement uncertainties.

Least-squares Method for $R_{V}$ and $A_{V}$. After deriving the probability of the reddening in a los, the most likely reddening values,

$$
E_{i}=(E(u-g), E(g-r), E(r-i), E(i-z)),
$$

can be obtained from the probability density function (PDF). They can then be used to derive the $R_{V}$ and $A_{V}$ given an extinction model, such as Cardelli et al. (1989) (hereafter $\mathrm{CCM}$ ), from the following equations,

$$
\begin{aligned}
& E(u-g)=\left(\left(a_{u}+\frac{b_{u}}{R_{V}}\right)-\left(a_{g}+\frac{b_{g}}{R_{V}}\right)\right) * A_{V}, \quad E(g-r)=\left(\left(a_{g}+\frac{b_{g}}{R_{V}}\right)-\left(a_{r}+\frac{b_{r}}{R_{V}}\right)\right) * A_{V} \\
& E(r-i)=\left(\left(a_{r}+\frac{b_{r}}{R_{V}}\right)-\left(a_{i}+\frac{b_{i}}{R_{V}}\right)\right) * A_{V}, \quad E(i-z)=\left(\left(a_{i}+\frac{b_{i}}{R_{V}}\right)-\left(a_{z}+\frac{b_{z}}{R_{V}}\right)\right) * A_{V} .
\end{aligned}
$$

These are linear equations for $A_{V}$ and $A_{V} / R_{V}$ and can be easily solved with a leastsquares or $\chi^{2}$ method to find the best fit $A_{V}$ and $R_{V}$ for each BHB star. The averaged $A_{V}$ and $R_{V}$ in each los are obtained from the median values of all the stars located in the los; and the uncertainties can be estimated from the median absolute deviation. The terms $a_{x}$ and $b_{x}$ are given from CCM.

Validation of the Methods. We used 900 Monte Carlo simulations to validate the Bayesian method, the left and middle panels in Fig. 1 show the comparison between the estimated and the true extinction values (given in the simulations) in two colors. The mean $1 \sigma$ error bars (less than $0.01 \mathrm{mag}$ ) are marked at the bottom, which suggests that the Bayesian method we employed in this work is robust.

To validate the least-squares method, we solve Eq. 2.6 for the $E(B-V)$ data looked up from the SFD extinction maps for each los and the fixed value of $R_{V}=3.1$. The right panel in Fig. 1 presents the histogram of $R_{V}$ in the simulation. The red curve is the Gaussian fit profile with the mean value $\left\langle R_{V}>\simeq 3.1\right.$ and $\sigma \simeq 0.16$, the yellow line marks the location of $R_{V}=3.1$. 

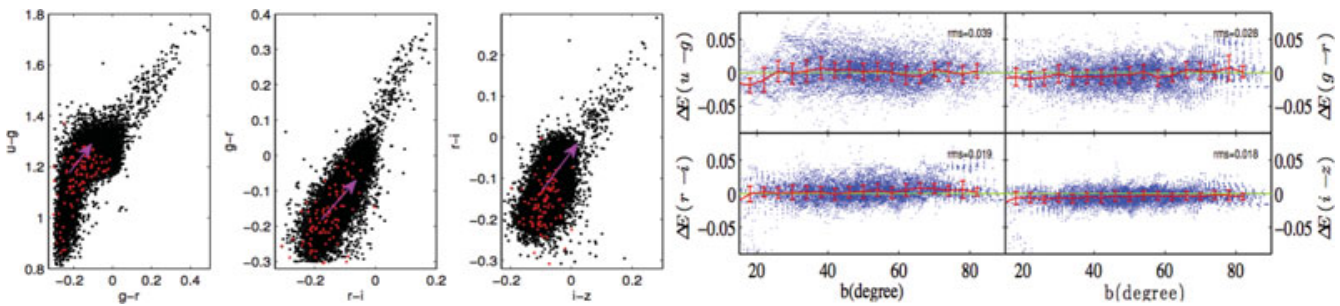

Figure 2. Sample distribution in the 2-color space, and the reddening contrasts with SFD.
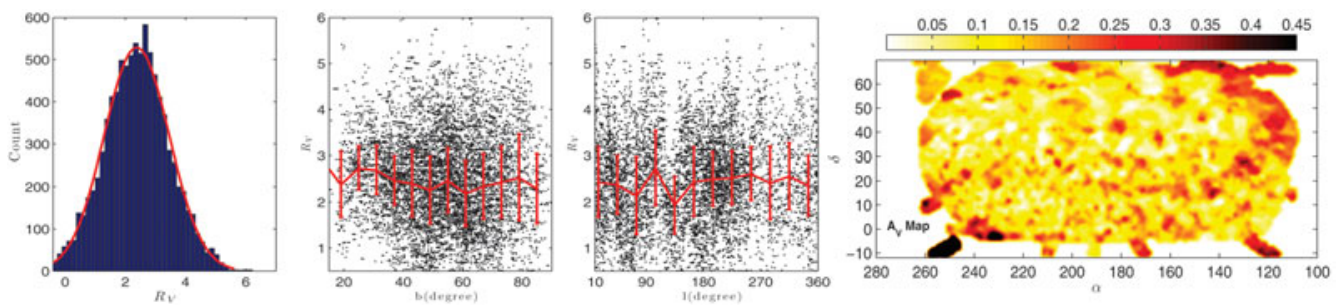

Figure 3. Distributions of the measured $R_{V}$ (the first three subplots), and $A_{V}$ map.

\section{Application to the BHB stars}

Data Selection. A total of 12530 field BHB stars are selected from Smith et al. (2010), as shown with the black points in the left panel of Fig. 2. The red points are the 94 zeroreddened template BHB stars selected from seven known globular clusters. The magenta arrows show the reddening direction.

Reddening Values. The reddening values estimated by Bayesian method are compared with SFD in the right panel of Fig. 2. They are well in agreement with each other.

$R_{V}$ and $A_{V}$ Values. The left panel in Fig. 3 shows the histogram distribution of the measured $R_{V}$, best-fitted by a Gaussian with $\mu \simeq 2.4$ and $\sigma \simeq 1.05$. The middle two are the measured $R_{V}$ as a function of the Galactic latitude (the second panel) and longitude (the right panel), respectively. The red curves show $\left\langle R_{V}\right\rangle$, which keeps constant at $\sim 2.5$ over all latitudes and longitudes. The right is the estimated $A_{V}$ map.

\section{Conclusions}

To measure the extinction, we propose a Bayesian method, and validated the method with simulations, which indicates accuracy is around $0.01 \mathrm{mag}$. It is robust even in the case that the reddening values are close to the observational errors. The extinctions derived from the SDSS BHB stars with this method are high consistent with SFD.

Acknowledgments. We thank the grants (No. U1231123, U1331202, U1231119, 11073024, 11 $\overline{103027, \text { U1331113 }}, 11303020)$ from NSFC and the support from LAMOST fellowship.

\section{References}

Arce, H. G. \& Goodman, A. A. 1999, ApJ, 512, L135

Cardelli, J. A., Clayton, G. C., \& Mathis, J. S. 1989, ApJ, 345, 245

Draine, B. T. 2003,ARA\& $A, 41,241$

Guy, J., Sullivan, M., Conley, et al. 2010, A\&A, 537, A7

Schlegel, D. J., Finkbeiner, D. P., \& Davis, M. 1998, ApJ, 500, 525

Smith, K. W., Bailer-Jones, C. A. L., Klement, R. J., \& Xue, X. X., 2010, A\&A, 522, A88

Tian, H. J., Neyrinck, M. C., Budavári, T., \& Szalay, A. S., 2011, ApJ, 728, 34T 